\title{
CHEMICAL COMFOSITION VARIATIONS IN THE GALAXY
}

\author{
Ju. L. FRANTSMAN \\ Radioastrophysical observatory \\ Latvian Academy of Sciences \\ Turgeneva 19, 226524, Riga, Latvia
}

ABSTRACT.Comparing the data about the Cepheid periods and the star evolution calculations, the conclusion is made that inside $5 \mathrm{kpc}$ distance from the Sun heavy element abundance $Z$ changes between 0.01 and 0.04 .

Most Cepheids are believed to be in the phase of core helium burning. Theoretical frequency-period distributions were obtained [1] by taking into account where, during its helium burning phase, each model of a series of models of different composition crosses the instability strip and how long it spends pulsating there. The results show the extreme sensitivity of the distribution to variations in the composition. These theoretical results agree with the observations of Cepheid metallicity [2] (Figure 1).

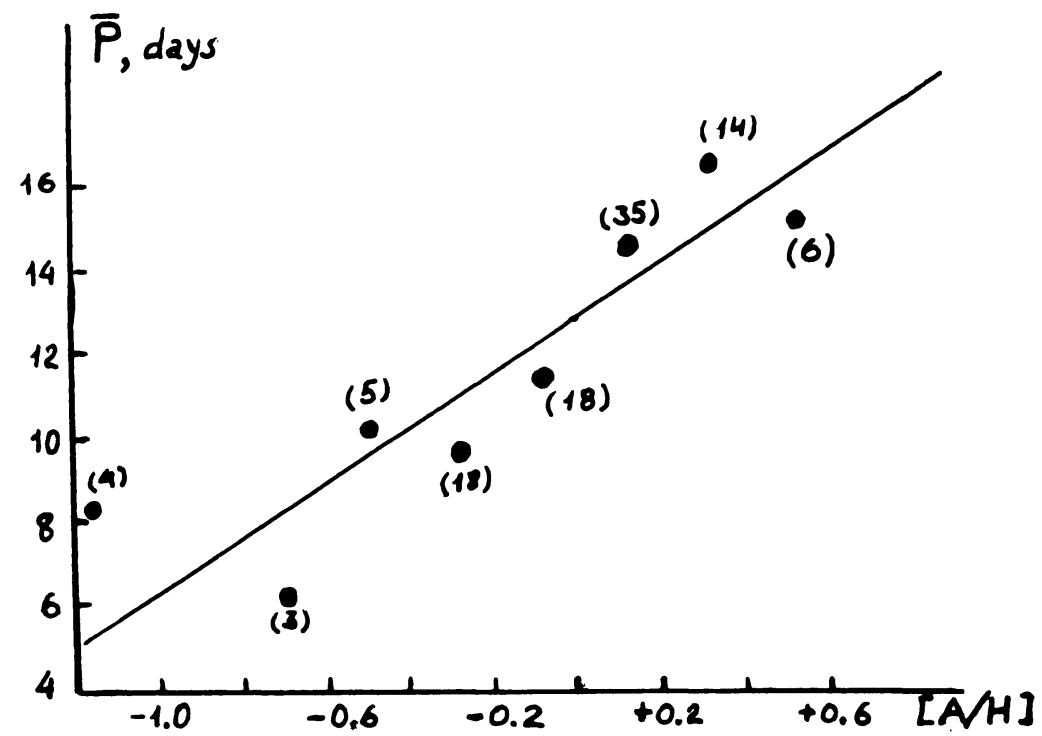

Figure 1. The observational mean period - metal abundance $\mathrm{A} / \mathrm{H}$ diagram. Numbers - the amount of Cepheids. 
In Figure 2 the mean period distributions are shown in rings on the different distances from the Sun. It is possible to distinguish regions with considerable amount of long period Cepheids: in the direction of the center of the Galaxy beginning with 2 kpc (apparantly due to the gradient of the heavy elements in the Galaxy), the regions with $1=70-90$ within 2-3 kpc of the Sun and $1=225-270$ within 3-4 kpC of the Sun. Comparing these observational data about Cepheid periods and the results obtained by the star evolution calculations (Figure 2 ) the conclusion is made that within $5 \mathrm{kpC}$ from the Sun the heavy element abundance $Z$ of matter changes between 0.01 and 0.04 .
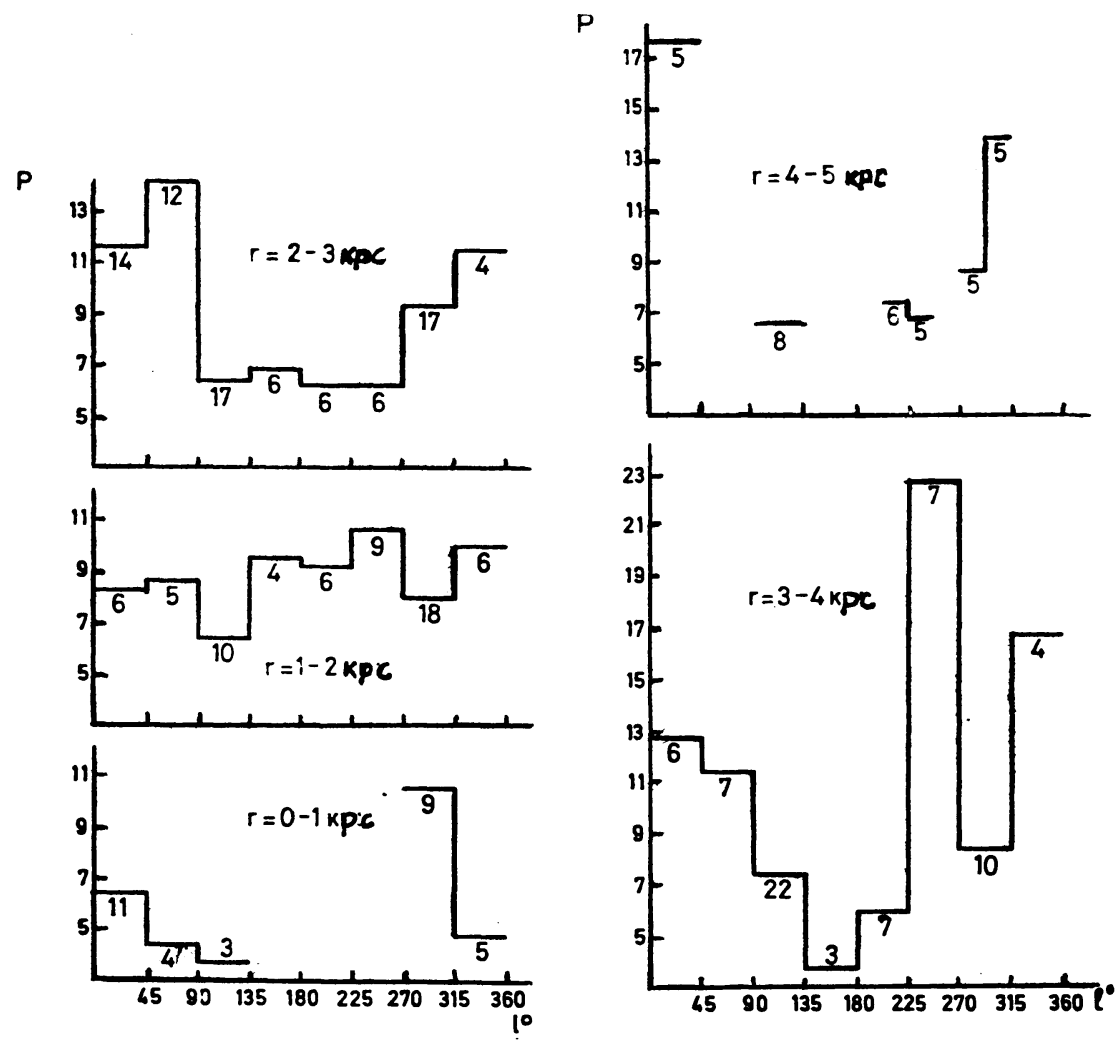

Figure 2. The mean period of Cepheids in the Galaxy distributions along the longitude in the rings on the different distances from the sun.

References.

1. Becker S.A., Iben I.,Jr., Tuggle R.S. (1977) "On the frequency-period distributions of Cepheid variables in galaxies in the local group", Ap. J., 218, 633 .

2. Harris H.C. (1981) 'Photometric abundances of classical cepheids and the gradient in the Galactic disk", A. J., 86, 707. 